IRSTI 29.15.17

https://doi.org/10.26577/phst.2021.v8.i2.02

\title{
Analytical determination of the non-relativistic quantum mechanical properties of near doubly magic nuclei
}

\author{
E. A. Thompson* iD, E. P. Inyang iD and E. S. William iD \\ Theoretical Physics Group, Department of Physics, University of Calabar, Calabar, Nigeria \\ *e-mail:edyy7000@yahoo.com
}

\begin{abstract}
A non-relativistic analytical study of some atomic nuclei near doubly-magic nuclei, namely $41 \mathrm{Ca}$ and $41 \mathrm{Sc}$. These nuclei under study are considered to have a closed core with a single nucleon orbiting the core, thus agreeing perfectly with the nuclear shell model. The superposition of Hulthen potential plus Spin - orbit interaction plus adjusted Coulomb is used as the nucleon - nucleon potential for this study. This superposition forms a suitable potential model useful in describing the net force mediating the interactions between nucleons in a nuclear system. The combined potentials effectively represent three basic interactions within the nucleus. By employing the Nikiforov - Uvarov method of solving the Schrodinger equation, we have calculated the energy eigenvalues and the eigen function of these nuclei. Also, the expectation values for radius, kinetic energy and momentum of each nucleus under study are also evaluated analytically. The results obtained are in agreement with experiment. As such, the constructed potential model used in the present work is recommended for similar nuclei.
\end{abstract}

Key words: Schrödinger equation, Hulthen potential, spin-orbit interaction, Coulomb potential, energy eigenvalues eigenfunction, expectation values.

\section{Introduction}

The atomic nuclei are an intricate quantum mechanical system. The study of its structure, which boarders on how its constituent particles and nucleons are arranged, is key to understanding and interpreting certain observations displayed by bulk matter. The atomic nucleus is made up of protons and neutrons that are arranged in a predictable order within the nuclear volume. [1-2]. A clear insight into the structure of the nuclear system, which is the core of nuclear properties research, coupled with our understanding of inter-nucleon forces, can be explored and used to critically test both our knowledge of the nuclear forces and many-body theories [3-4].

The shell model and the single - particle energies offer a veritable ground for study and understanding basic features and properties of atomic nuclei [5-8]. The single-particle energies obtained using the nuclear shell model are expected to be influenced by the nuclear spin-orbit force [9]. However, the shell model is aptly applicable to closed shells with a lone valence-nucleon nuclei [10]. Most importantly, the single-particle behaviour is observed in near magic and near doubly magic nuclei, where the major shells are filled up with nucleons in a nucleus, leaving a single proton or neutron to occupy the next shell after a major or minor gap. For instance, ${ }^{41} \mathrm{Ca}$ and ${ }^{41} \mathrm{Sc}$ are modelled as doubly magic nuclei with a single particle orbiting the $1 f_{\frac{7}{2}}$ level. The shell model predicts that the properties of a nucleus are correctly represented by the properties of the lone nucleon orbiting the core, taking the pairing effect into consideration [11]. As such, a valence proton orbiting the $\mathrm{N}=\mathrm{Z}$ $=20$ core for the ${ }^{41} \mathrm{Sc}$ isotope and a valence neutron orbiting the $\mathrm{N}=\mathrm{Z}=20$ core for the ${ }^{41} \mathrm{Ca}$ are used to predict to a large extent accurately the properties of these nuclei.

In this study, we have constructed a nuclear potential model within the non-relativistic domain of quantum mechanics which takes into consideration the basic interactions taking place within the atomic nucleus. These interactions include the strong interaction represented in our model by the Hulthen potential, the spin-orbit interaction represented here by the spin-orbit potential and the electromagnetic interaction 
represented in our model by the Coulomb potential. By the superposition of these three potential models, we have been able to model the forces mediating the basic interactions within the nucleus. As such, our constructed potential model is suitably applied to the Schrodinger wave equation to obtain the energy eigenvalues, wave function and other quantum mechanical observables for the nucleus.

The Hulthen potential is a central mean field potential used to present a short-ranged force field. In this research, the Hulthen represents the strong force which influence the interaction between nucleons inside the nucleus. In the present study, the Hulthen potential is the nucleon - nucleon $(\mathrm{N}-\mathrm{N})$ potential. The spin-orbit interaction is a vital component of the force field under which nucleons interacts [12]. The spin-orbit effect arises from the interaction of the nucleon magnetic moment with the mean field potential. This effect is usually visible for orbits with $\ell \geq 0$ [13]. Hence, coupling the spinorbit interaction to the mean field potential for nucleon - nucleon interaction is of essence. In relativistic quantum mechanics, the spin is effectively represented in the wave equation (Dirac equation) describing the motion of a spin $1 / 2$ particle [14]. Although the non-relativistic Schrodinger wave equation does not consider the spin of a particle rightly, in this study the spin has been factored in by introducing the spin-orbit interaction into the N-N potential. This has been done successfully in this work, as is presented in the subsequent sections. Interactions between the core, which contains the positively charged proton, and the orbiting valence proton necessitate the introduction of the adjusted Coulomb potential.

In this study, the non-relativistic nuclear shell model is used to obtain analytically the single particle ground state energies for ${ }^{41} \mathrm{Ca}$ and ${ }^{41} \mathrm{Sc}$, near doubly magic nuclei. To achieve this aim, we solved analytically the Schrodinger equation for a constructed potential model formed by the superposition of the Hulthen potential [15] plus spin-orbit potential plus the adjusted Coulomb potential [16] as an effective potential between the core and a single particle. These potentials are important nuclear potentials used in describing the interaction between a single nucleon and the nuclear core. [17-20]. However, the adjusted Coulomb potential strength is set to zero in the case where the single particle orbiting the core is a neutron. Furthermore, the Hellman-Feynman theorem is used to obtain some physical observables such as radii, kinetic energies, and momenta for the nuclei under study.

Now that the choice of the N-N potential has been made, the next step is to solve the Schrodinger wave equation. We use the Nikiforov-Uvarov (NU) method to solve the Schrodinger equation [21]. The organization of this paper is as follows: The NU method is reviewed in section 2; the non-relativistic energy spectrum is presented in section 3; expectation values of physical observables are stated in section 4, and results and discussion are presented in section 5 .

\section{Review of the NU method}

We introduce the NU method briefly in this section; details can be obtained from ref. [21]. This method is based on solving the second order differential equation of the hyper geometric type:

$$
\psi^{\|}(z)+\frac{\tilde{\tau}(z)}{\sigma(z)} \psi^{\prime}(z)+\frac{\widetilde{\sigma}(z)}{\sigma^{2}(z)} \psi(z)=0
$$

In Eq. (1), $\sigma(z)$ and $\tilde{\sigma}(z)$ are polynomials of at most second degree. $\tilde{\tau}(z)$ is a first-degree polynomial, and $\psi(z)$ is the hypergeometric-type function, $\psi^{\| l}(z)$ and $\psi^{\prime}(z)$ are the second and the first order derivative of the hypergeometric- type function with respect to the spatial coordinates respectively. A possible solution to Eq. (1) is proposed as

$$
\psi(z)=\phi(z) y(z)
$$

The function $y(z)$ which is the second part of the proposed solution given by Eq. (2) can be obtained from the equation below;

$$
\sigma(z) y^{\| \prime}(z)+\tau(z) y^{\mid}(z)+\lambda y(z)=0
$$

Equation (3) is the hypergoemetric type second order differential equation obtained from the relationship between the polynomials $\sigma(z)$ and $\tilde{\tau}(z)$, details of this relationship can be obtained from Nikiforov and Uvarov ref. [21].

From Eq. (2), the function $\phi(z)$ is the solution of a differential equation of the form given by:

$$
\sigma(z) \phi^{\mid}(z)-\pi(z) \phi(z)=0
$$

where

$$
\tau(z)=\breve{\tau}(z)+2 \pi(z)
$$


and $\lambda$ in Eq. (3) is a parameter defined as

$$
\begin{aligned}
\lambda=\lambda_{n} & =-n \tau^{\mid}(z)+\frac{n(n-1)}{2} \sigma^{\mid} \mid(z)= \\
& =0(n=0,1,2,3, \ldots)
\end{aligned}
$$

The function $\tau(z)$ is a polynomial in terms of $z$, and to obtain a proper solution, its first derivative $\tau^{\mid}(z)$ must be negative. The function $y(z)$ as stated in Eq. (2) is the hyper geometric type wave function obtained by using the Rodrigues relation:

$$
y_{n}(z)=\frac{B_{n}(z) d^{n}}{\rho(z) d z^{n}}\left[\sigma^{n}(z) \rho(z)\right]
$$

where $B_{n}$ is a constant related to normalization, and the weighted function $\rho(z)$ is defined as:

$$
\frac{d}{d z}[\sigma(z) \rho(z)]=\tau(z) \rho(z)
$$

Also, the function $\pi(z)$ which is a first-degree polynomial is defined as

$$
\begin{array}{r}
\pi(z)=\frac{\sigma^{\mid}(z)-\tilde{\tau}(z)}{2} \pm \\
\pm \sqrt{\left(\frac{\sigma^{\mid}(z)-\tilde{\tau}(z)}{2}\right)^{2}-\tilde{\sigma}(z)+k \sigma(z)}
\end{array}
$$

$\mathrm{k}$ in Eq. (9) is related to the parameter $\lambda$ Eq. (6), and the first derivative of $\pi^{\mid}(z)$ as

$$
\lambda=k+\pi^{\mid}(z)
$$

The value of $\mathrm{k}$ is obtained by equating the discriminant of the quadratic expression under the square root sign in Eq. (10) to zero, and solving the resulting equation for $\mathrm{k}$. By solving Eq. (6) and Eq. (10), we derive the energy eigenvalue equation.

\section{Solution of Schrodinger equation for} Hulthen potential plus spin-orbit interaction plus adjusted Coulomb potential

The eigenvalues of the energy $\mathrm{E}$ for nuclei is obtained by solving the radial component of the 3dimensional Schrodinger wave equation given as

$$
\begin{gathered}
\frac{d^{2} R(r)}{d r^{2}}+\frac{2}{r} \frac{d R(r)}{d r}+ \\
+\frac{2 \mu}{\hbar^{2}}\left[E_{n \ell j}-V_{e f f}(r)\right] R(r)=0
\end{gathered}
$$

This equation is for a single particle, where $\mu$ is the reduced mass of a single nucleon, $E_{n \ell j}$ is the energy spectrum, $V_{\text {eff }}$ is our constructed potential model which has in it the centrifugal term, $\hbar$ is the Planck's constant, $n, \ell$ and $\mathrm{j}$ are the principal, orbital and total momentum quantum number respectively. By transforming the variable from $r \rightarrow z$, let a new variable be defined as

$$
z=e^{-\alpha r}
$$

The derivatives of the function $R(r)$ in Eq. (11) are obtained in terms of the new variable $\mathrm{z}$, as such Eq. (11) is transformed and restated in terms of $z$ as $R(r)$ is redefined as $\psi(z)$.

By transforming Eq. (11) using the new variable defined by Eq. (12), the Schrodinger equation becomes as stated below

$$
\frac{d^{2} \psi(z)}{d z^{2}}+\frac{1}{z} \frac{d \psi(z)}{d z}+\frac{1}{\alpha^{2} z^{2}}\left[\frac{2 \mu E}{\hbar^{2}}-\frac{2 \mu}{\hbar^{2}}\left(V_{e f f}\right)\right]=0
$$

The central potential in this case is the Hulthen potential which is an approximation of the wellknown Woods Saxon potential. This potential has been successfully used to approximate the nuclear central force:

$$
V_{H}(r)=-\frac{V_{o} \alpha e^{-\alpha r}}{1-e^{-\alpha r}}
$$

where $V_{o}$ in Eq. (14) is the potential strength of the Hulthen potential.

The adjusted Coulomb potential is given as

$$
V_{c}(r)=\frac{e^{2}}{R_{o}}\left[3-\left(\frac{r}{R_{o}}\right)^{2}\right]
$$

where $\mathrm{e}$ is the charge of $\mathrm{e}^{-}$or proton, $\mathrm{R}_{0}$ the radius of nuclei and $r$ is the range of this position of a particle from the core. The spin-orbit interaction is given as

$$
V_{L S}(r)=\frac{1}{2} V_{L S}(0)\left(\frac{r_{o}}{\hbar}\right)^{2} \frac{1}{r}\left[\frac{d V_{H}(r)}{d r}\right] \vec{L} . S
$$

where $\vec{L} \cdot \vec{S}=\frac{\hbar^{2}}{2}\left(j(j+1)-\ell(\ell+1)-\frac{3}{4}\right)$.

The centrifugal term of the Schrodinger wave equation is given as

$$
V_{c f}=\frac{\hbar^{2}}{2 \mu} \frac{\ell(\ell+1)}{r^{2}}
$$


The effective potential $\mathrm{V}_{\text {eff }}$ for the nuclei system is obtained by summing up Eq. (14), Eq. (15), Eq. (16) and Eq. (17) as given by Eq. (18):

$$
\begin{gathered}
V_{e f f}=-\frac{V_{o} e^{-\alpha r}}{1-e^{-\alpha r}}- \\
-V_{L S}(0) r_{o}^{2} \frac{1}{r} \frac{\alpha e^{-\alpha r}}{\left(1-e^{-\alpha r}\right)^{2}} \vec{L} \cdot \vec{S}- \\
-\frac{3 e^{2}}{\pi \varepsilon_{0} R_{o}}+\frac{e^{2} r^{2}}{\pi \varepsilon_{0} R_{o}^{3}}+\frac{\hbar^{2}}{2 \mu} \frac{\ell(\ell+1)}{r^{2}}
\end{gathered}
$$

We apply the Greene-Aldrich approximations scheme [22], [23], [24] to handle the centrifugal barrier. The approximation is valid for $\alpha \ll 1$. It is of the following forms:

$$
\begin{aligned}
\frac{1}{r^{2}} & \approx \frac{4 \alpha^{2} e^{-2 \alpha r}}{\left(1-e^{-2 \alpha r}\right)^{2}} \\
\frac{1}{r^{2}} & \approx \frac{\alpha^{2}}{\left(1-e^{-2 \alpha r}\right)^{2}}
\end{aligned}
$$

The approximations stated in Eq. (19) and Eq. (20) are commonly used in calculations, but in the present work Eq. (20) is used, as this particular approximation scheme suit our purpose. Applying the Greener-Aldrich approximation scheme as stated by Eq. (20) to solve the Schrodinger equation makes our solution an approximate solution. An exact solution can only the attempted for our constructed potential if $\ell=0$, but this will cancel the spin-orbit effect from the results. Putting Eq. (18), Eq. (19) and Eq. (20) into Eq. (13), the Schrodinger equation now reads:

$$
\frac{d^{2} \psi(z)}{d z^{2}}+\frac{1}{z} \frac{d \psi(z)}{d z}+\frac{1}{z^{2}(1-z)^{2}}\left[\begin{array}{l}
\frac{2 \mu E}{\hbar^{2} \alpha^{2}}(1-z)^{2}+V_{o} \frac{2 \mu}{\hbar^{2} \alpha^{2}} z(1-z) \\
+V_{L S}(0) \frac{2 \mu r_{o}^{2}}{\hbar^{2}}\left(\frac{z}{1-z}\right)\left(j(j+1)-\ell(\ell+1)-\frac{3}{4}\right) \\
-\frac{6 \mu e^{2}}{R_{o} \pi \varepsilon_{0} \alpha^{2} \hbar^{2}}(1-z)^{2}+\frac{2 \mu e^{2}}{\hbar^{2} \pi \varepsilon_{0} \alpha^{4} R_{o}^{3}}(1-z)^{4} \\
-\ell(\ell+1) z
\end{array}\right] \psi(z)=0
$$

By applying the principles suggested by [25] into the higher powers of $\mathrm{z}$ beyond the second degree, Eq.
(21) is transformed to the hyper geometric-type second order differential equation solvable by the NU method:

$$
\frac{d^{2} \psi(z)}{d z^{2}}+\frac{(1-z)}{z(1-z)} \frac{d \psi(z)}{d z}+\frac{1}{z^{2}(1-z)^{2}}\left[\begin{array}{l}
-\frac{2 \mu E}{\hbar^{2} \alpha^{2}} z^{2}+V_{L S}(0) \frac{2 \mu r_{o}^{2}}{\hbar^{2}}\left(j(j+1)-\ell(\ell+1)-\frac{3}{4}\right) z^{2} \\
-\frac{6 \mu e^{2}}{R_{o} \pi \varepsilon_{0} \alpha^{2} \hbar^{2}} z^{2}+6 \frac{2 \mu e^{2}}{\hbar^{2} \pi \varepsilon_{0} \alpha^{4} R_{o}^{3}} z^{2}-V_{o} \frac{2 \mu}{\hbar^{2} \alpha} z^{2} \\
+2 \frac{2 \mu E}{\hbar^{2} \alpha^{2}} z+V_{L S}(0) \frac{2 \mu r_{o}^{2}}{\hbar^{2}}\left(j(j+1)-\ell(\ell+1)-\frac{3}{4}\right) z \\
+2 \frac{6 \mu e^{2}}{R_{o} \pi \varepsilon_{0} \alpha^{2} \hbar^{2}} z-4 \frac{2 \mu e^{2}}{\hbar^{2} \pi \varepsilon_{0} \alpha^{4} R_{o}^{3}} z-\ell(\ell+1) z \\
+V_{o} \frac{2 \mu}{\hbar^{2} \alpha} z-\frac{2 \mu E}{\hbar^{2} \alpha^{2}}-\frac{6 \mu e^{2}}{R_{o} \pi \varepsilon_{0} \alpha^{2} \hbar^{2}}+\frac{2 \mu e^{2}}{\hbar^{2} \pi \varepsilon_{0} \alpha^{4} R_{o}^{3}}
\end{array}\right] \psi(z)=0
$$

where

$$
\begin{gathered}
\varphi=\left\{\begin{array}{l}
-\frac{2 \mu E}{\hbar^{2} \alpha^{2}}+V_{L S}(0) \frac{2 \mu r_{o}^{2}}{\hbar^{2}}\left(j(j+1)-\ell(\ell+1)-\frac{3}{4}\right) \\
-\frac{6 \mu e^{2}}{R_{o} \pi \varepsilon_{0} \alpha^{2} \hbar^{2}}+6 \frac{2 \mu e^{2}}{\hbar^{2} \pi \varepsilon_{0} \alpha^{4} R_{o}^{3}}-V_{o} \frac{2 \mu}{\hbar^{2} \alpha}
\end{array}\right\} \\
\eta=\left\{\begin{array}{l}
2 \frac{2 \mu E}{\hbar^{2} \alpha^{2}}+V_{L S}(0) \frac{2 \mu r_{o}^{2}}{\hbar^{2}}\left(j(j+1)-\ell(\ell+1)-\frac{3}{4}\right) \\
+2 \frac{6 \mu e^{2}}{R_{o} \pi \varepsilon_{0} \alpha^{2} \hbar^{2}}-4 \frac{2 \mu e^{2}}{\hbar^{2} \pi \varepsilon_{0} \alpha^{4} R_{o}^{3}}+V_{o} \frac{2 \mu}{\hbar^{2} \alpha}-\ell(\ell+1)
\end{array}\right\}
\end{gathered}
$$




$$
-\mathfrak{R}=\frac{2 \mu E}{\hbar^{2} \alpha^{2}}+\frac{6 \mu e^{2}}{R_{o} \pi \varepsilon_{0} \alpha^{2} \hbar^{2}}-\frac{2 \mu e^{2}}{\hbar^{2} \pi \varepsilon_{0} \alpha^{4} R_{o}^{3}}
$$

Substituting equations (23), (24) and (25) into Eq. (22), we have:

$$
\begin{array}{r}
\frac{d^{2} \psi(z)}{d z^{2}}+\frac{(1-z)}{z(1-z)} \frac{d \psi(z)}{d z}+ \\
+\frac{1}{z^{2}(1-z)^{2}}\left[\varphi z^{2}+\eta z-\mathfrak{R}\right] \psi(z)=0
\end{array}
$$

Comparing (26) and (1), the relevant polynomials can be expressed as

$$
\begin{gathered}
\tilde{\tau}(z)=1-z \\
\sigma(z)=z(1-z), \quad \sigma^{2}(z)=z^{2}(1-z)^{2} \\
\tilde{\sigma}(z)=\varphi z^{2}+\eta z-\mathfrak{R}
\end{gathered}
$$

Inserting the polynomials as defined by Eq. (27) into Eq. (9), we have:

$$
\pi(z)=\frac{-z}{2} \pm \sqrt{(\gamma-k) z^{2}+(\eta+k) z+\Re}
$$

where

$$
\gamma=\frac{1}{4}+\varphi
$$

As required by the NU method the discriminant of the quadratic expression under the square root sign in Eq. (28) is equated to zero. This leads to another quadratic equation in terms of $\mathrm{k}$. Solving the ensuing quadratic equation for $\mathrm{k}$, we have possible solutions as:

$$
k=-(\eta+2 \Re) \pm 2 \sqrt{\mathfrak{R}} \sqrt{\gamma+\eta+\Re}
$$

Accepting the negative solution of $\mathrm{k}$ from Eq. (30), and substituting k into Eq. (28), we have

$$
\pi(z)=\frac{-z}{2} \pm(\sqrt{\mathfrak{R}}+\sqrt{\gamma+\eta+\mathfrak{R}) z}-\sqrt{\mathfrak{R}}
$$

Upon inserting Eq. (31) into Eq. (5) another required polynomial $\tau(\mathrm{z})$ is derived:

$$
\begin{gathered}
\tau(z)=1-2 z- \\
-2(\sqrt{\mathfrak{R}}+\sqrt{\gamma+\eta+\mathfrak{R}}) z-2 \sqrt{\Re}
\end{gathered}
$$

Taking the first derivative of Eq. (32), we have:

$$
\tau^{\mid}(z)=-2-2 \sqrt{\mathfrak{R}}-2 \sqrt{\gamma+\eta+\Re}
$$

Taking the first derivative of Eq. (31) and using Eq. (30), Eq. (33) as well as Eq. (27), $\lambda$ and $\lambda_{n}$ are obtained explicitly as

$$
\begin{gathered}
\lambda=\frac{-1}{2}-\sqrt{\Re}-\sqrt{\gamma+\eta+\Re}- \\
-\eta-2 \Re-2 \sqrt{\Re} \sqrt{\gamma+\eta+\Re} \\
\lambda_{n}=n^{2}+n+2 n \sqrt{\Re}+2 n \sqrt{\gamma+\eta+\Re}
\end{gathered}
$$

By comparing Eq. (34) and Eq. (35), we obtain the quantity $\mathfrak{R}$ :

where

$$
\mathfrak{R}=\left[\frac{-\frac{1}{2}\left[\left(n+\frac{1}{2}+\sqrt{\beta}\right)^{2}+\frac{1}{4}+\xi\right]}{\left(n+\frac{1}{2}+\sqrt{\beta}\right)}\right]^{2}
$$

$$
\begin{array}{r}
\beta=\gamma+\eta+\Re \\
\xi=-\frac{1}{4}-\gamma+\Re
\end{array}
$$

Reintroducing Eq. (23), Eq. (24) and Eq. (25) into Eq. (37) and Eq. (36), we obtain the energy eigenvalues for the superposition Hulthen potential, spin-orbit interaction and adjusted Coulomb potential in terms of the principal $n$, orbital $\ell$ and angular momentum $\mathrm{j}$ quantum numbers as stated by Eq. (38): 


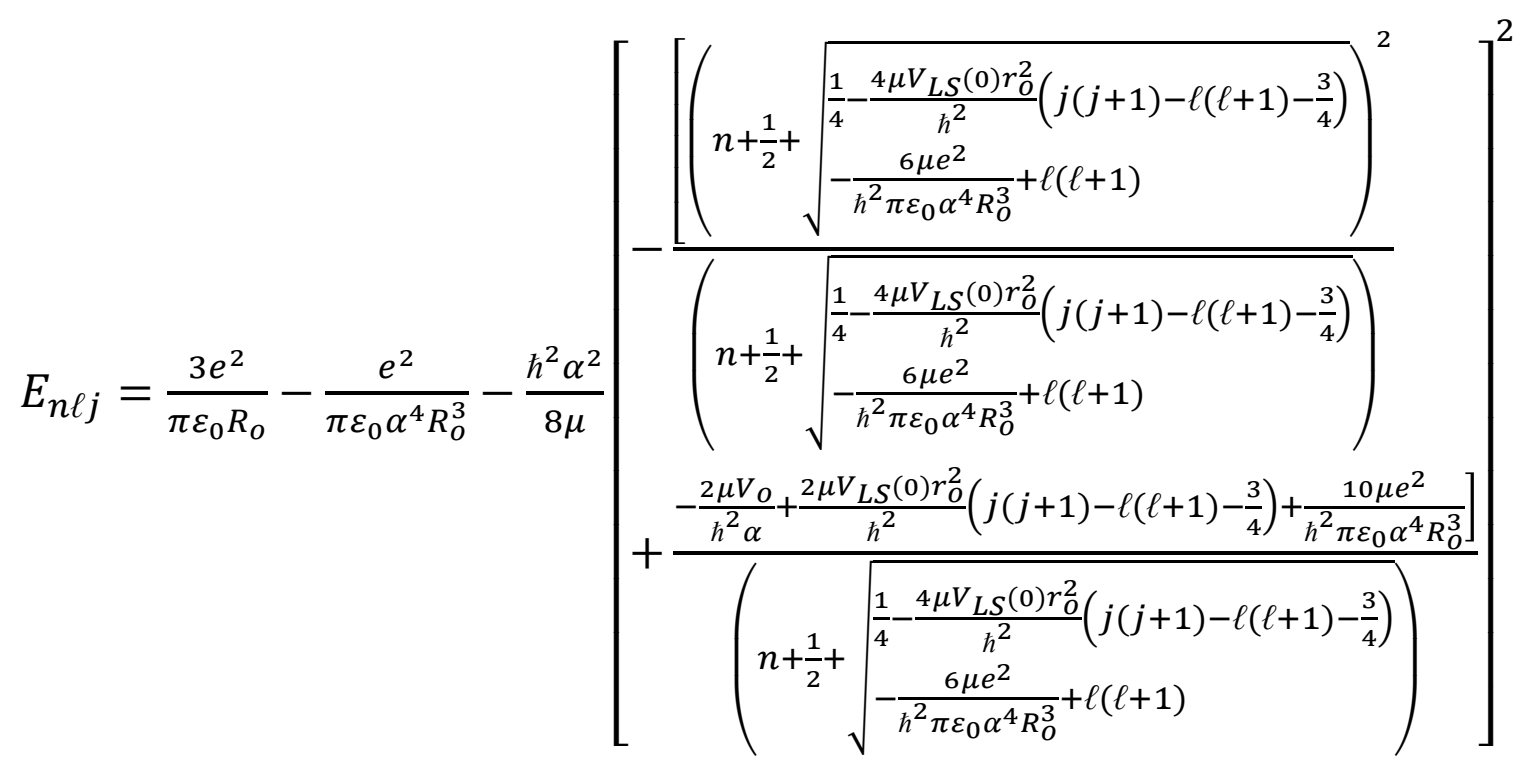

Equation (38) is the energy eigenvalue equation for the proton orbiting the core in a nucleus. The energy spectrum for a neutron orbiting the core of the nucleus is obtained by setting e in Eq. (38) to zero as the net charge of the neutron is zero, as there is no Coulomb interaction between the orbiting neutron and the core of the nucleus. Hence the energy eigenvalues for the neutron is given as:

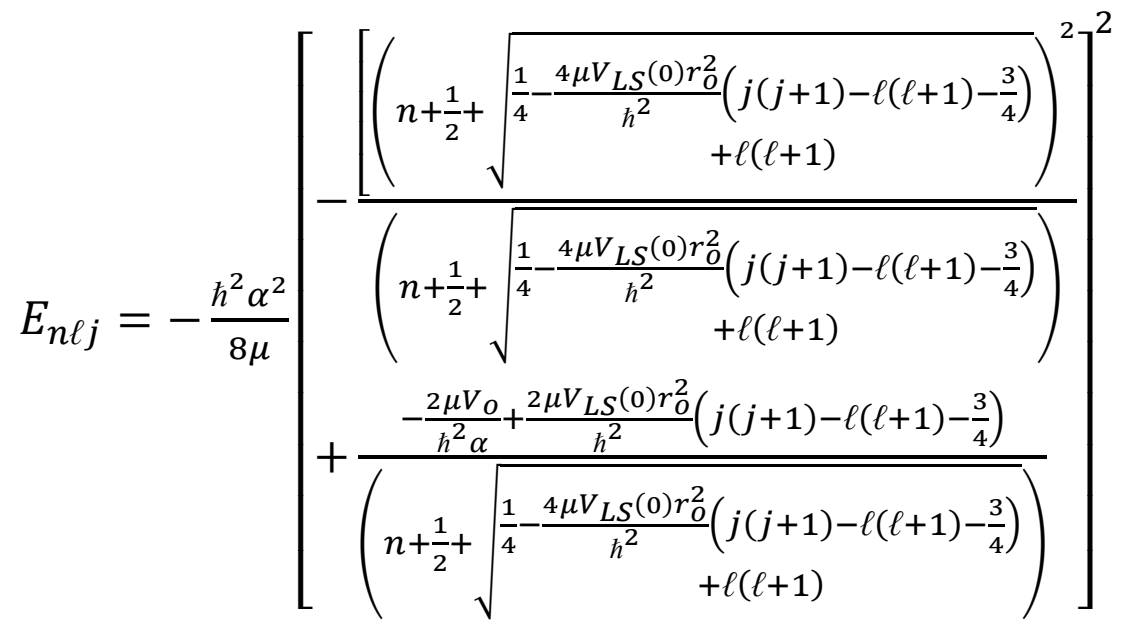

Equation (38) and Eq. (39) are used in this study to obtain explicitly the ground state energy of ${ }^{41} \mathrm{Sc}$ and ${ }^{41} \mathrm{Ca}$ respectively.

Next, we obtain the wave function as stated in Eq. (8), using the weighted factor $\rho(z)$ which is in the form

$$
\rho(z)=z^{2 \sqrt{\Re}} \cdot(1-z)^{2 \sqrt{\gamma+\eta+\Re}}
$$

Substituting Eq. (40) into Eq. (7), the Rodrigues relation is obtained as:

$$
\begin{gathered}
y_{n}(z)=B_{n} z^{-2 \sqrt{\Re}}(1-z)^{-2 \sqrt{\gamma+\eta+\Re}} \frac{d^{n}}{d z^{n}}\left[z^{n+2 \sqrt{\Re}}(1-z)^{n+2 \sqrt{\gamma+\eta+\Re}}\right] \equiv \\
\equiv P_{n}^{(2 \sqrt{\Re}, 2 \sqrt{\gamma+\eta+\Re)}}(1-2 z)
\end{gathered}
$$


where $P_{n}$ is the Jacobi polynomial. Using Eq. (36) and Eq. (41) the wave function is given as:

$$
\begin{gathered}
\psi(z)=N_{n \ell} z^{\sqrt{\Re}}(1- \\
-z)^{\left(\frac{1}{2}+\sqrt{\gamma+\eta+\Re}\right)} P_{n}^{(2 \sqrt{\Re}, 2 \sqrt{\gamma+\eta+\Re)}(1-2 z)}
\end{gathered}
$$

where $N_{n \ell}$ is the normalization constant. Applying the normalization condition the normalization constant is obtained as:

$$
\frac{N_{n \ell}^{2}}{\alpha} \int_{-1}^{1}\left(\frac{1-y}{2}\right)^{\omega}\left(\frac{1+y}{2}\right)^{b}\left[P_{n}^{(\omega, b-1)} y\right]^{2} d y=1
$$

Equation (43) is derived by setting

$$
\begin{gathered}
b=\frac{1}{2}+\sqrt{\gamma+\eta+\mathfrak{R}} \\
\omega=2 \sqrt{\mathfrak{R}}
\end{gathered}
$$

By comparing Eq. (43) to the standard integral of the form Eq. (37) as reported by Ebomwonyi et at., in the ref. [24], we have:

$$
\begin{gathered}
\int_{-1}^{1}\left(\frac{1-p}{2}\right)^{x}\left(\frac{1+p}{2}\right)^{y}\left[P_{n}^{(2 x, 2 y-1)} y\right]^{2} d p= \\
=\frac{2 \Gamma(x+n+1) \Gamma(y+n+1)}{n ! x \Gamma(x+y+n+1)}
\end{gathered}
$$

We derive the normalization constant as:

$$
N_{n \ell}=\left[\frac{\alpha n ! \omega \Gamma(\omega+b+n+1)}{2 \Gamma(\omega+n+1) \Gamma(b+n+1)}\right]^{\frac{1}{2}}
$$

\section{Expectation values of some physical} observables

Now we derive the expectation values for position (radius), kinetic energy and the square of momentum for the respective nuclei under study. To achieve this, we use the Hellmann-Feynman theorem. 'The Hellmann-Feynman theorem assures that a non-degenerate eigenvalue of a hermitian operator in a parameter dependent eigensystem varies with respect to the parameter provided that the associated normalized eigenfunction is continuous with respect to the parameter' [26]. This statement is given mathematically as:

$$
\left\langle\psi_{\mathrm{q}}\left|\frac{\partial \mathrm{H}}{\partial \mathrm{q}}\right| \psi_{\mathrm{q}}\right\rangle=\frac{\partial \mathrm{E}}{\partial \mathrm{q}}
$$

The Hamiltonian used in solving the Schrodinger equation for the present work is of the form:

$$
\begin{gathered}
H=-\frac{\hbar^{2}}{2 \mu} \frac{d^{2}}{d r^{2}}- \\
-\frac{\hbar^{2}}{\mu r} \frac{d}{d r}+\frac{\hbar^{2}}{2 \mu r^{2}} \ell(\ell+1)- \\
-\frac{V_{o} e^{-\alpha r}}{1-e^{-\alpha r}}- \\
-V_{L S}(0) \frac{r_{o}^{2} \alpha e^{-\alpha r}}{\left(1-e^{-\alpha r}\right)^{3}} \vec{L} \cdot \vec{S}
\end{gathered}
$$

To obtain the expectation value for position of the particle in the nuclear system, which represent approximately the radius of the nuclei under study, we equate the variable q in Eq. (47) to $\ell$. Substituting Eq. (48) into Eq. (47), we have:

$$
\left\langle\psi(\ell)\left|\frac{\partial H}{\partial \ell}\right| \psi(\ell)\right\rangle=\frac{\hbar^{2}}{2 \mu}(2 \ell+1)\left\langle r^{-2}\right\rangle
$$

where $\left\langle r^{-2}\right\rangle$ is the expectation value for position (radius of the system).

Taking the derivative of Eq. (39) with respect to $\ell$ as required by Eq. (47), we have:

$$
\frac{\partial E}{\partial \ell}=\left[\begin{array}{l}
-2 A Z\left[(2 \ell+1)\left(\frac{4 \mu V_{\ell S} r_{O}^{2}}{\hbar^{2}}+1\right)\right] . \\
\left\{\frac{1}{2 \sqrt{y}}-\frac{\frac{1}{2 \sqrt{y}}\left(\frac{2 \mu V_{0}}{\hbar^{2} \alpha}+\frac{2 \mu V_{\ell S} r_{O}^{2}}{\hbar^{2}}\left(j(j+1)-\ell(\ell+1)-\frac{3}{4}\right)+\frac{10 \mu e^{2}}{\hbar^{2} \pi \varepsilon_{o} \alpha^{2} R_{o}^{3}}\right)}{\left(n+\frac{1}{2}+\sqrt{y}\right)^{2}}\right\}
\end{array}\right]
$$

Equating (49) and (50), the expectation value for position is derived as: 


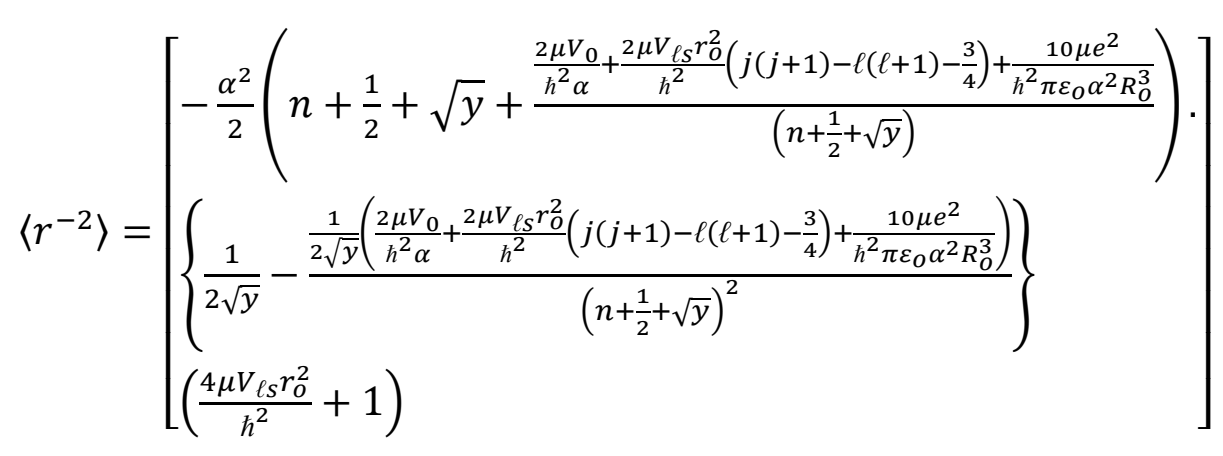

The expectation value for position (radius) $\langle r\rangle$ is obtained from Eq. (51). The expectation value for kinetic energy $\langle T\rangle$ is obtained from Eq. (47) by setting

$\mathrm{q}$ to $\mu$, and differentiating the Hamiltonian and the energy spectrum with respect to the reduced mass $\mu$ respectively. We have the derivatives below:

$$
\frac{\partial H}{\partial \mu}=-\frac{1}{\mu}\left(-\frac{\hbar^{2}}{2 \mu} \frac{d}{d r^{2}}-\frac{\hbar^{2}}{\mu r} \frac{d}{d r}+\frac{\hbar^{2}}{2 \mu r^{2}} \ell(\ell+1)\right)(
$$

Recall that,

$$
\begin{gathered}
H=T+V \\
T=H-V
\end{gathered}
$$

where $\mathrm{T}$ is the kinetic energy and $\mathrm{V}$ the potential, putting Eq. (53) into Eq. (52) we have:

$$
\left\langle\psi(\mu)\left|\frac{\partial H}{\partial \mu}\right| \psi(\mu)\right\rangle=-\frac{1}{\mu}(H-V)=-\frac{1}{\mu}\langle T\rangle(54)
$$

Also,

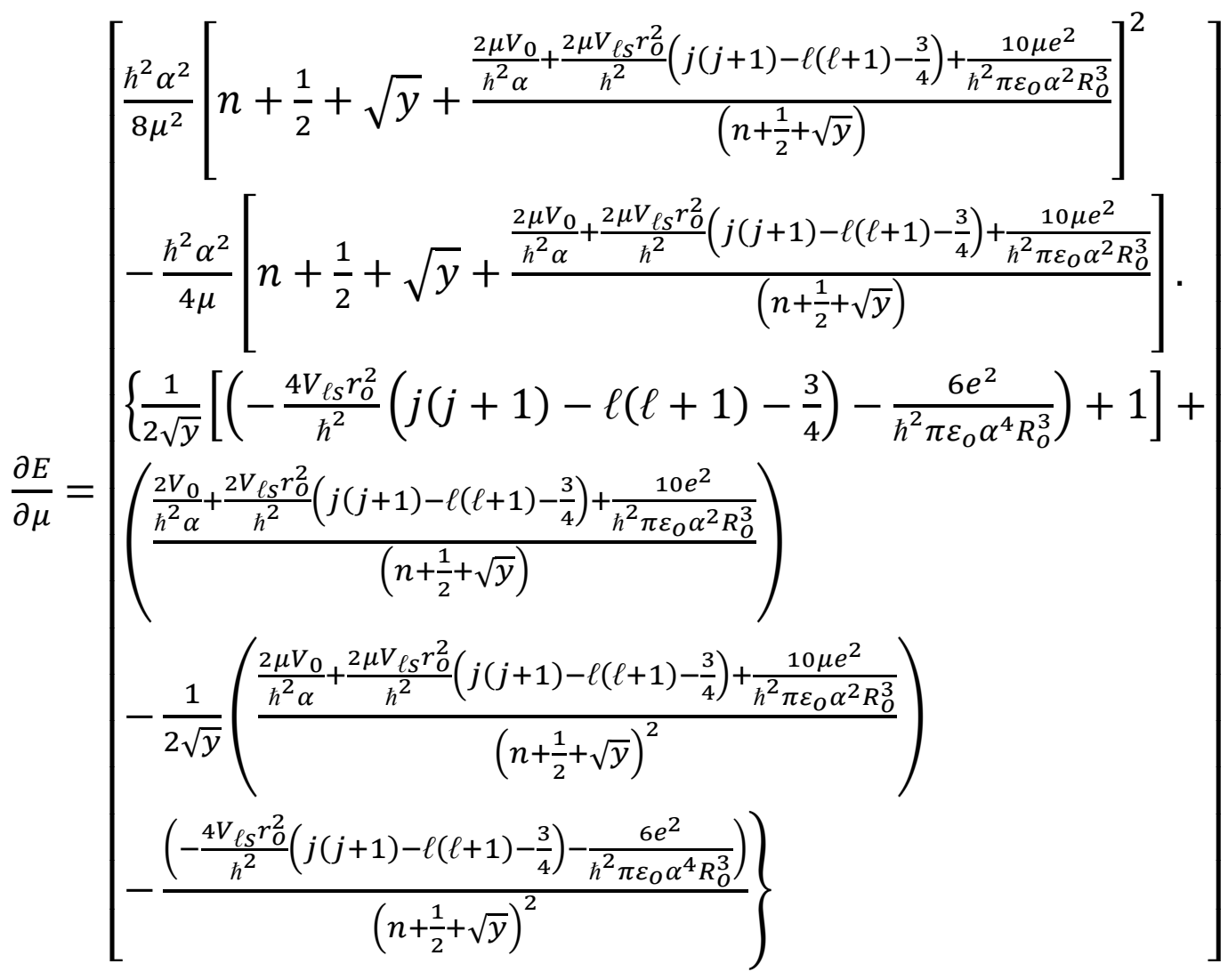


Equating (54) and (55) as required by (47), and we obtain the expectation value for kinetic energy as:

$$
\langle T\rangle=\left[\begin{array}{l}
\frac{\hbar^{2} \alpha^{2}}{8 \mu}\left[n+\frac{1}{2}+\sqrt{y}+\frac{\frac{2 \mu V_{0}}{\hbar^{2} \alpha}+\frac{2 \mu V_{\ell S} r_{O}^{2}}{\hbar^{2}}\left(j(j+1)-\ell(\ell+1)-\frac{3}{4}\right)+\frac{10 \mu e^{2}}{\hbar^{2} \pi \varepsilon_{O} \alpha^{2} R_{O}^{3}}}{\left(n+\frac{1}{2}+\sqrt{y}\right)}\right] \\
-\frac{\hbar^{2} \alpha^{2}}{4}\left[n+\frac{1}{2}+\sqrt{y}+\frac{\frac{2 \mu V_{0}}{\hbar^{2} \alpha}+\frac{2 \mu V_{\ell S} r_{O}^{2}}{\hbar^{2}}\left(j(j+1)-\ell(\ell+1)-\frac{3}{4}\right)+\frac{10 \mu e^{2}}{\hbar^{2} \pi \varepsilon_{O} \alpha^{2} R_{O}^{3}}}{\left(n+\frac{1}{2}+\sqrt{y}\right)}\right.
\end{array}\right] .
$$

Using the relationship between kinetic energy and the square of momentum as:

$$
-\frac{1}{\mu}\langle T\rangle=-\frac{1}{2 \mu^{2}}\left\langle P^{2}\right\rangle
$$

The square of momentum is obtained from Eq. (57) as stated below: 


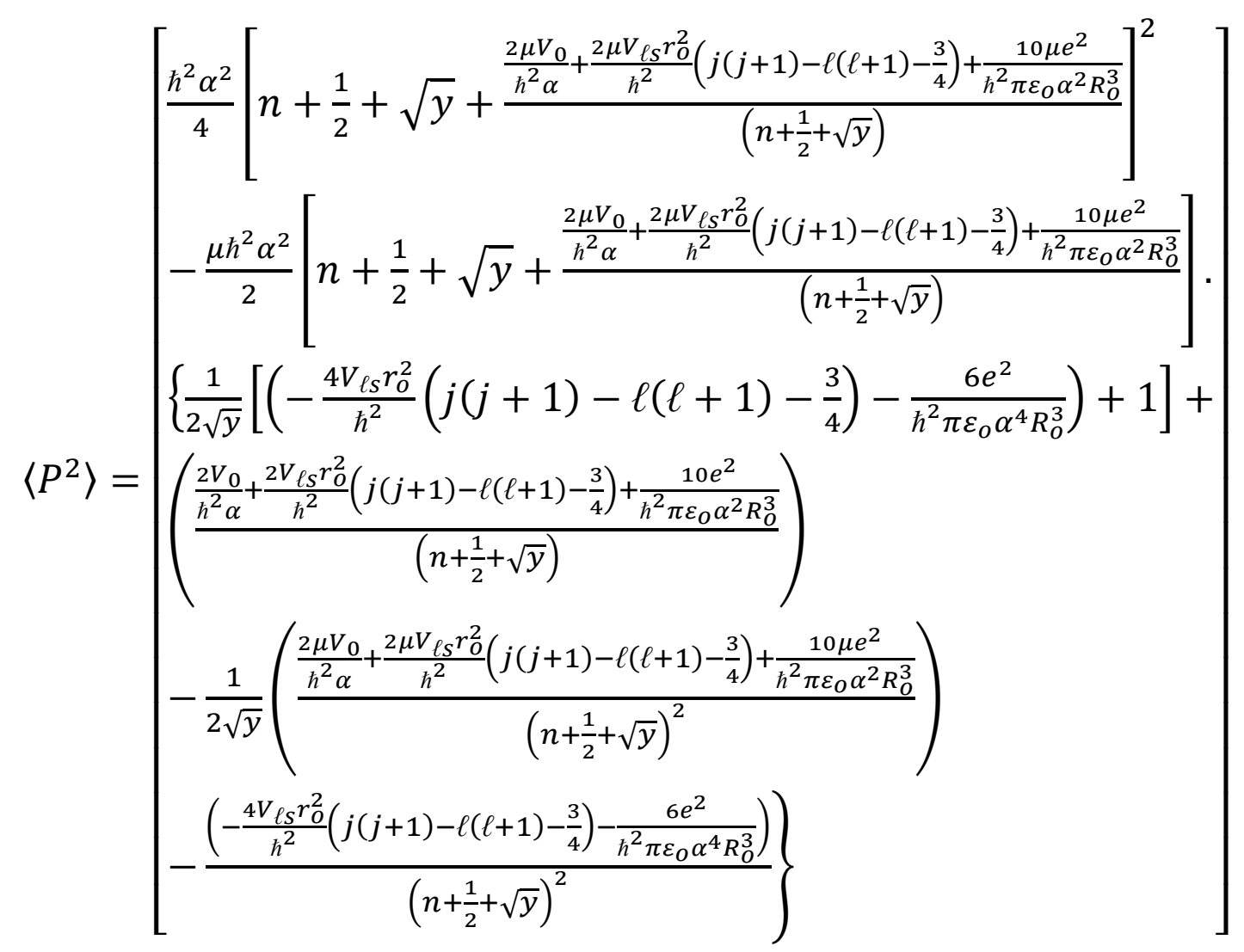

\section{Results and discussion}

The analytical solution of the Schrodinger wave equation for the superposition of Hulthen potential, spin-orbit interaction and adjusted Coulomb potential is applied to study two nuclides; calcium 41 and scandium 41 isotopes.

Explicitly: ${ }^{41} \mathrm{Ca}$ and ${ }^{41} \mathrm{Sc}$ nuclei are chosen as nuclear systems in this study, being that the best evidence of single particle behavior is easily observed in near doubly magic nuclei. The nucleus of ${ }^{41} \mathrm{Ca}$ has a closed shell for both nucleons with a valence neutron in the $1 f_{\frac{7}{2}}$ level. Also, ${ }^{41} \mathrm{Sc}$ has closed shells for protons and neutrons, with a valence proton in the $1 f_{\frac{7}{2}}$ level. The ground state properties of ${ }^{41} \mathrm{Ca}$ and ${ }^{41} \mathrm{Sc}$ are determined by the valence nucleon orbiting the closed core according to the theory of nuclear shell model. We obtain the numerical results of the ground state energies of ${ }^{41} \mathrm{Ca}$ and ${ }^{41} \mathrm{Sc}$ from Eq. (39) and Eq. (38) respectively. Table 1 gives a summary of the parameters used for the numerical calculation. The numerical results of these energies of the nuclei under study are as presented in Table 2 and compared to experimental data culled from ref [13]. The calculated energy levels agree to a very great extent with the experimental values. Comparing the ground state energy of ${ }^{41} \mathrm{Ca}$ obtained from this study to the experimental value shows a percentage relative error of $2.02 \%$. The calculated ground state energy of ${ }^{41} \mathrm{Sc}$ obtained from the present work shows a percentage relative error of only $0.95 \%$ to that obtained experimentally. The small percentage relative error recorded here attests to the success of the analytical method employed in this work as well as the excellent means of adding the spin orbit interaction to the Hulthen potential plus the Coulomb potential, which serves as the nuclear potential for the nuclei under study. The physical observables such as the expectation values for position (radius), kinetic energy, and the square of momentum are as presented on Table 3. Using the same set of parameters used for calculating the numerical values for the energies of both nuclei under study, Eq. (51) was used to obtain the numerical values for the position (radius) of the nuclei, Eq. (56) was used to obtain the kinetic energies and Eq. (58) was used to obtain the numerical values for the square momentum of both nuclei. Comparing the 
calculated expectation value for the radius of the isotopes under study to the experimental values of their radii showed a relative percentage error of $20.8 \%$. The numerical values for kinetic energy and momentum obtained from this work were not compared to experimental values of these quantities owing to the unavailability of experimental data on these quantities for the isotopes under study.

Table 1 - Numerical values of potential parameters for the combined potential

\begin{tabular}{|c|c|c|}
\hline Parameters & ${ }^{41} \mathrm{Ca}$ & ${ }^{41} \mathrm{Sc}$ \\
\hline $\boldsymbol{\alpha}\left(\mathrm{fm}^{-1}\right)$ & 0.5 & 0.5 \\
$\boldsymbol{V}_{\boldsymbol{o}}(\mathrm{MeV})$ & 45.83 & 45.83 \\
$\boldsymbol{V}_{\boldsymbol{L S}}(\mathrm{MeV})$ & 20.1652 & 20.1652 \\
$\hbar \boldsymbol{c}(\mathrm{MeV})$ & 197.3269602 & 197.3269602 \\
$\boldsymbol{\mu}\left(\mathrm{MeV} / \mathrm{c}^{2}\right)$ & 915.20392928 & 915.212583522 \\
$\boldsymbol{R}_{\boldsymbol{o}}(\mathrm{fm})$ & 4.1378607 & 4.178607 \\
$\boldsymbol{\varepsilon}_{\boldsymbol{0}}$ & 0.079785 & 0.079785 \\
$\boldsymbol{r}_{\boldsymbol{o}}$ & 0.2 & 0.2 \\
$\mathrm{e}(\mathrm{MeV})$ & - & 1.199999999 \\
\hline
\end{tabular}

Table 2 - Ground state energy of ${ }^{41} \mathrm{Ca}$ and ${ }^{41} \mathrm{Sc}$

\begin{tabular}{|c|c|c|c|}
\hline Nuclides & State & $\begin{array}{c}\text { Calculated Energy }(\mathrm{MeV}) \\
\text { \{present work }\end{array}$ & $\begin{array}{c}\text { Experimental value of } \\
\text { Energy }(\mathrm{MeV})[23]\end{array}$ \\
\hline $\mathbf{4 1} \boldsymbol{C} \boldsymbol{a}$ & $1 f_{\frac{7}{2}}$ & -357.51025 & -350.4148 \\
$\mathbf{4 1 S c}$ & $1 f_{\frac{7}{2}}$ & -346.41183 & -343.1371 \\
\hline
\end{tabular}

Table 3 - Numerical results of the expectation values for radius, kinetic energy and square of momentum of ${ }^{41} \mathrm{Sc}$ and ${ }^{41} \mathrm{Ca}$ isotopes

\begin{tabular}{|c|c|c|c|}
\hline \multicolumn{2}{|c|}{ Quantity } & ${ }^{41} \mathbf{S c}$ & ${ }^{41}$ Ca \\
\hline \multirow{2}{*}{ Radius $\left\langle\boldsymbol{r}^{2}\right\rangle^{\frac{1}{2}}(\mathrm{fm})$} & Calculated value & 2.74591 & 2.75152 \\
\cline { 2 - 4 } & $\begin{array}{c}\text { Experimental value (Angeli } \\
\text { et al) }\end{array}$ & 3.4698 & 3.4780 \\
\hline \multirow{2}{*}{ Kinetic energy $\langle\boldsymbol{T}\rangle(\mathrm{eV})$} & Calculated value & 342.86341 & 337.22125 \\
\cline { 2 - 4 } & Experimental value & - & - \\
\hline \multirow{2}{*}{ Square of momentum $\left\langle\boldsymbol{P}^{2}\right\rangle$} & Calculated value & $6.27580 \times 10^{5}$ & $6.17251 \times 10^{5}$ \\
\cline { 2 - 4 } & Experimental value & - & - \\
\hline
\end{tabular}

\section{Conclusions}

In this study, we obtained the approximate solution of the Schrodinger wave equation for the superposition of Hulthen potential with spin-orbit potential plus adjusted Coulomb potential using the NU method. Clearly, we calculated the single particle energy spectrum for this constructed potential model and applied it to obtain the ground state energy of near doubly magic nuclei, namely,
${ }^{41} \mathrm{Ca}$ and ${ }^{41} \mathrm{Sc}$. We also obtained the single particle normalized wave function for the nuclei under study. Using the Hellman-Feynman theorem, the physical observables such as the radius, kinetic energy and momentum of selected near doubly magic nuclei were calculated in this study. Therefore, we propose that our constructed potential model can be used to study nuclei such as near doubly magic and near singly magic nuclei. 


\section{References}

1 A. De-Shalit, I. Talimi, Nuclear shell theory. Weizmann Institute of Science, Rehovoth, Isreal, 1962.

2 M. G. Mayer, J. H. D. Jensen, Elementary theory of nuclear shell structure, Wiley, New York, 1955.

3 B. R. Barrett, D. J. Dean, M. Hjorth-Jensen, J. P. Vary. Nuclear forces and the quantum many-body problem // Journal of physics G. Nuclear and particle physics. - 2005. - Vol. 31. - P. 8. https://doi.org/10.1088/09543899/31/8/E01

4 B. L. Cohen, I. B. L. Cohen. Concepts of Nuclear Physics, McGraw-Hill Companies, New York, USA, 1971.

5 M. A. Preston, R. K. Bhaduri. Structure of the nucleus, CRC Press, 2018.

6 H. Heylen. Ground state properties near the $\mathrm{N}=20$ and $\mathrm{N}=40$ Islands of inversion. PhD dissertation, MPG Germany, 2016.

7 S. G. Nilson, I Ragnarsson. Shapes and shells in nuclear structure, Cambridge University Press, Cambridge, $100,1995$.

8 S. Bogner, T. T. S. Kuo, L. Coraggio, A. Covello, N. Itaco. Low momentum nucleon -nucleon potential and shell model effective interactions // Phys.Rev.C. $-2002 . \quad$ - Vol. 65. - Pp. 1-5. https://doi.org/10.1103/PhysRevC.65.051301

9 A. Nikman, A. A. Rajabi, M. Solaimani. Solution of D-dimensional Schrodinger equation for Woods-Saxon potential with spin-orbit, Coulomb and centrifugal terms through a new hydrid numerical fitting Nikiforov- Uvarov method // J. Theor. Appl. Phys. -2016. -Vol. 10. -No.1. -Pp. 53-59.

10 K. S. Krane. Introductory nuclear physics $3^{\text {rd }}$ ed., Wiley and Sons Inc., New York, 1988.

11 P. Goldhammer. The structure of light nuclei // Review of modern physics. - 1963. - Vol. 35. - No. 1. - P. 40. https://doi/10.1103/ RevModPhys.35.40

12 H. J Mang, H. A. Weidenmuller. Shell-model theory of the nucleus // Annual Rev. Nucl. Sci. - 1968. - Vol. 18. 1. - Pp. 1-26. https:///doi/ abs/10.1146/annurev.ns.18.120168.000245

13 M. Mousavi, M. R. Shojaei. Calculation of energy and charge radius for doubly-magic nuclei of ${ }^{41} \mathrm{Ca}$ and ${ }^{41} \mathrm{Sc}$ with extra nucleon // Chinese journal of physics. -2016. -Pp. 1-6.

14 R. Sever, C. Tezcan, O. Yesiltas, M. Bucurgat. Exact solution of effective mass Schrodinger equation for the Hulthen potential // Int. J. Theor. Phys. - 2008. - Pp. 2243-2248. https://doi/10.1007/s10773-008-9656-7

15 S. M. Ikhdair, R. Sever. Approximate eigenvalue and eigenfunction solutions for the generalized Hulthen potential with any angular momentum // Journal of Mathematical Chemistry. -2007. - Vol. 42. - No.3. - Pp. 461-471. https://doi/10.1007/s10910-006-9115-8

16 J. Hughes, K. J. Le Couteur. Spin-orbit coupling in the nuclear shell model, Department of theoretical Physics, monograph, University of Liverpool, 1950.

17 E. S. William, E.P. Inyang, E. A. Thompson, Arbitrary 1 - solution of the Schrodinger equation interacting with Hulthen-Hellman potential model // Rev. Mex. Fis. - 2020. - Vol.66. - No.6. - Pp. 730-741. https://doi.org/10.13349/ RevMesFis.66.730

18 C. O. Edet, U. S. Okorie, A. T. Ngiangia, A. N. Ikot. Bound state solution of the Schrodinger equation for the modified Kratzer potential plus screened Coulomb potential // Indian J. Phys. -2020. -Vol. 94. -Pp. 425-433. https://doi.org/10.26850 /1678-4618eqj.v45.1.2020.p65-77

19 M. R. Pahlavani, S. A. Alavi. Solution of Woods-Saxon potential with spin-orbit and centrifugal terms through Nikiforov-Uvarov method// Comm. Thoer. Phys. -2012. -Vol. 58. -No.5. -Pp. 739-743. http://dx.doi.org/10.1088/0253$6102 / 58 / 5 / 19$

20 A. F. Nikiforov, V.B. Uvarov. Special function of mathematical physics, Birkhauser, Basel, 1988.

21 R. L. Greene, C. Aldrich. Variational wave function for screened Coulomb potential // Rev. A. -1976. - Vol. 14. - No. 6. - P. 2363. https://doi.org/10.1103/physRev.14.2363

22 S. H. Dong, W. C. Qiang, G. H. Sun, V. R. Bezerra. Analytical approximation to the 1-wave solution of the Schrodinger equation with the Eckart potential // J. phys. A.: Mathematical \& Theoretical. -2007. - Vol. 40. - No.34. - Pp. 1-7.

23 C. A. Ebomwonyi, M. C. Onate, M. C. Onyeaju, A. N. Ikot. Any 1 state solution of the Schrodinger equation interacting with Hellmann generalized Morse potential model // Karbala Intl. J. Mod. Sc. - 2017. - Vol. 3. - No.1. Pp. 1-10. http://dx.doi.org/10.1016/j.kijoms.2017.03.001

24 A. Tas, O. Aydogdu, M. Salti. Relativistic spinless particle with position dependent mass: Bound state and scattering phase shift // J. Korean Phys. Soci. -2017. - Vol.70. - No.10. - Pp. 896-904. https://doi:10.1007/s12648-02001908-y

25 D. Wallace. An introduction to Hellmann-Feynman theory, M. Sc. Thesis, Department of Mathematics, University of Central Florida, Orlando, USA, 2005.

26 I. Angeli, K. P. Marinova, Table of experimental nuclear state charge radii: An update, atomic data and nuclear data table. - 2013. - Vol. 99. - No. 1. - Pp. 69-95. https://doi.10.1016/j.adt.2011.12.006 